\title{
Impact of Blade Mounting Structures on Cross-Flow Turbine Performance
}

\author{
Benjamin Strom, ${ }^{1}$ a) Noah Johnson, ${ }^{1}$ and Brian Polagye ${ }^{1}$ \\ Dept. of Mechanical Engineering, University of Washington Box 352600, NE Stevens Way, Seattle, WA, 98195, \\ $U S A$
}

(Dated: 24 April 2018)

Cross-flow or vertical-axis turbines are flow energy conversion devices in which lift forces cause blades to rotate around an axis perpendicular to the flow. In marine currents, rivers, and some wind energy applications, cross-flow turbines are a promising alternative to more conventional axial-flow turbines. The performance implications of the choice of structure used to mount turbine blades to the central shaft is examined experimentally in a recirculating water flume. Turbine performance is found to be strongly dependent on choice of mounting structure. Power loss due to rotational drag on these structures is estimated experimentally by rotating the mounting structure without blades. Through a perturbation-theory approach, interactions between turbine blades and mounting structures are examined. Analytical models for the power loss due to mounting structure drag are introduced and shown to be consistent with experiments. To provide guidance for cross-flow turbine design, the models are re-formulated in terms of non-dimensional turbine design and operational parameters. Mounting blades solely at their mid-span is shown to decrease performance through multiple fluid effects. Using foil cross-section struts located at the turbine blade tips is found to result in the highest turbine performance.

Keywords: Cross-flow turbine, vertical-axis turbine, VAWT, end plates, blade mounting

\section{INTRODUCTION}

Cross-flow turbines convert fluid kinetic energy to rotational mechanical energy via blades rotating about an axis perpendicular to free stream direction. These turbines may be further sub-divided into those that generate torque from lift on foils (e.g., Darrieus rotors) and those that generate torque from drag (e.g., Savonius rotors). Despite historical concerns of low performance and structural failure due to fatigue, lift-based cross-flow turbines have experienced a resurgence of research and commercial interest in recent years and hold promise for urban $^{1}$ and offshore ${ }^{2}$ wind applications. Modern experimental and computational techniques have enabled substantial increases in power performance ${ }^{3}$ and suggestions that optimized arrays may be able to extract more power per area than arrays of axial-flow turbines ${ }^{4}$. Drag-based cross-flow turbines are also an area of active research ${ }^{5}$, but are not the focus of this investigation and recent developments (e.g., Plourde et al. ${ }^{6}$ ) are not discussed here. In addition to wind applications, cross-flow turbines have several features that make them a promising alternative to axial-flow (horizontal-axis) turbines when operated in marine or river currents. First, their typically rectangular form factor is well-suited for the geometry of shallow tidal and river channels, allowing for the construction of higher blockage ratio turbine arrays that could boost array performance ${ }^{7}$. Second, the maximum blade velocity of cross-flow turbines is generally lower than equivalently sized axial-flow turbines, reducing the risk of blade cavitation and potential harm to aquatic fauna through collision or acoustic emissions ${ }^{8}$. Third, cross-flow turbine

\footnotetext{
a)strombw@uw.edu
}

operation is either bi- or omni-directional, depending on the axis orientation eliminating the need for active yaw control in oscillating tidal currents.

In their simplest form, the rotor of a cross-flow turbine consists of a set of fixed-pitch blades that rotate about a central axis. Despite this single degree of freedom, the cyclically varying flow encountered by the blades, including interaction with their own wake, result in complex hydrodynamics. Studies have been conducted on the performance implications of various blade geometric parameters ${ }^{9-13}$, operating conditions ${ }^{14-17}$, and wake interactions $^{18}$. However, little has been published concerning the implications of how the blades are affixed to the central shaft of the turbine. While this concern may seem secondary to the rotor blade geometry, the blade support structure has the potential to heavily influence rotor performance. First, since the support member(s) must rotate with the rotor, they will necessarily produce some drag opposing the direction of rotation. Second, the support members may influence lift generation of the rotor blades. For example, on stationary foils, end-plates have been shown to be functionally similar to an increase in blade span by reducing tip effects ${ }^{19}$. Blade support structures placed near the tips of the blades may act as end-plates, reducing lift losses due to flow from the pressure surface to the suction surface around the end of the blade. Third, on aircraft wings, winglets are employed to reduce induced drag (drag due to lift ${ }^{20}$ ). Blade support structures have the potential to provide this function. Finally, differing blade support geometries may restrict span-wise flow in the turbine rotor to varying degrees, which may impact turbine performance by altering vortex formation during dynamic stall.

Previous studies have individually demonstrated several strategies for mounting the blades to the central shaft. These include disks ${ }^{21,22}$, streamlined struts at the 
blade ends ${ }^{23,24}$, and streamlined struts at the mid-span or other intermediate positions ${ }^{25,26}$. However, to date, few studies have made a systematic comparison of the implication of these choices. Goude, Lundin, and Leijon ${ }^{27}$ considered the influence of differing numbers of struts using a double-multiple stream tube model based on tabulated values of the strut drag coefficient. Gosselin, Dumas, and Boureau ${ }^{28}$ used an unsteady Reynolds-averaged Naiver-Stokes model to explore the effect of the adding end-plates to the blade tips of a cross-flow turbine. Small end-plate resulted in turbine performance gains, since reduction in tip-losses outweighed end-plate drag losses. Rawlings et al. ${ }^{29}$ experimentally found a slight increase in performance by applying disk and foil end-plates to the ends of a three-bladed cross-flow turbine. Li and Calisal $^{30}$ experimentally compared a turbine with NACA 0012 struts positioned at the center-span and ends of the blades to a more blunt profile, positioned at $1 / 4$ span from the ends. The increase in performance of the NACA 0012 struts was attributed to lower profile drag and a reduction in tip losses and induced drag. Additionally, disk and foil end-plates were tested resulting in a small increase in performance. Bachant et al. ${ }^{16}$ experimentally compared NACA 0021 and cylindrical struts mounted at the mid-span of a three bladed cross-flow turbine. The large drag on the cylindrical struts resulted in a negative turbine efficiency at all operating conditions. Strut drag was measured independently from turbine performance by rotating the turbines without blades.

This study experimentally explores the impact of blade mounting geometries on the performance of a lift-based cross-flow turbine with two straight blades. By evaluating changes in turbine performance and drag on mounting structures, as well as analytical models, we provide a guide for selecting blade mounting geometry for crossflow turbine designs. These considerations are specific to lift-based cross-flow turbines since drag-based crossflow turbines, such as a Savonius rotor, are configured to maximize drag.

\section{METHODS}

Cross-flow turbine performance is characterized by the tip-speed ratio $(\lambda)$ and rotor mechanical efficiency $\left(C_{P}\right)$, which are given by

$$
\lambda=\frac{\omega R}{U_{\infty}}
$$

and

$$
C_{P}=\frac{\omega \tau}{\frac{1}{2} \rho U_{\infty}^{3} 2 R H}
$$

where $\omega$ is the turbine rotation rate, $R$ is the turbine radius, $\rho$ is the operating fluid density, $U_{\infty}$ is the freestream velocity, $\tau$ is the torque produced by the turbine, and $H$ is the turbine height.
Experiments were performed in a recirculating water flume with a test section $75 \mathrm{~cm}$ wide and $47 \mathrm{~cm}$ deep. The turbines were $17.2 \mathrm{~cm}$ in diameter and $23.4 \mathrm{~cm}$ high, resulting in a constant blockage ratio of $11.4 \%$. Since only relative changes in turbine performance are considered, performance is not corrected for blockage. All tests used a turbine rotor with two, straight, NACA 0015 blades with a chord length of $4.06 \mathrm{~cm}$, resulting in a chord to radius ratio of 0.47 and a solidity,

$$
\sigma=\frac{N c}{2 \pi R},
$$

of 0.15 , where $N$ is the number of turbine blades. The blades were mounted at a preset pitch angle of 6 degrees, leading edge rotated outwards about the quarter-chord. Free-stream velocity measurements were made using an acoustic Doppler velocimeter at a sample rate of $64 \mathrm{~Hz}$. The turbulence intensity (standard deviation of turbulent fluctuations relative to the mean flow) was $1.5 \%$ at the maximum flow velocity of $0.7 \mathrm{~m} / \mathrm{s}$. For a given tipspeed ratio, the turbine angular velocity was held constant by a servomotor. Electrical power generated by the turbine, actualized as reverse current in the servomotor, was dissipated in a dump resistor. Angular velocity control, rather than constant torque control, was used to eliminate the confounding factor of changes in rotor mass moment of inertia for different blade mounting structures. The torque generated by the turbine rotor was measured using a six-axis load cell between the servomotor and a fixed mounting surface (Fig. 1). Turbine position was measured using a $2^{18}$ counts per revolution encoder. The lower end of the turbine shaft was mounted to the flume bottom via a bearing and a second six-axis load cell which measured parasitic bearing torque. For each test configuration, turbine forces, torque, and position were sampled at $1 \mathrm{kHz}$ for 30 seconds.

The torque used to calculate the mechanical rotor efficiency in Eq. (2) is the torque produced by the rotor due to fluid forcing only, $\tau=\tau_{\text {fluid }}$. When operated at constant angular velocity, the equation of motion for the turbine rotor is

$$
\tau_{\text {fluid }}+\tau_{\text {bearing }}+\tau_{\text {control }}=J \dot{\omega}=0,
$$

where $J$ is the mass moment of inertia of the turbine system and $\dot{\omega}$ is the angular acceleration. $\tau_{\text {bearing }}$ is the torque applied due to friction in the lower bearing, while $\tau_{\text {control }}$ is the torque applied to the turbine rotor by the servomotor and is the sum of electrical forces and bearing losses within the servomotor. Figure 1 illustrates these torques and the reaction torques measured in these experiments. The upper load cell measures the reaction torque of the servomotor, $\tau_{\mathrm{m} \text {, top }}$, equal and opposite $\tau_{\text {control }}$. The lower load cell measures the reaction torque on the the lower bearing, $\tau_{\mathrm{m}}$, bottom, equal and opposite

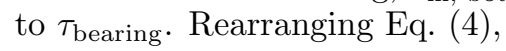

$$
\tau=-\tau_{\text {bearing }}-\tau_{\text {control }}=\tau_{\mathrm{m}, \text { top }}+\tau_{\mathrm{m}, \text { bottom }}
$$




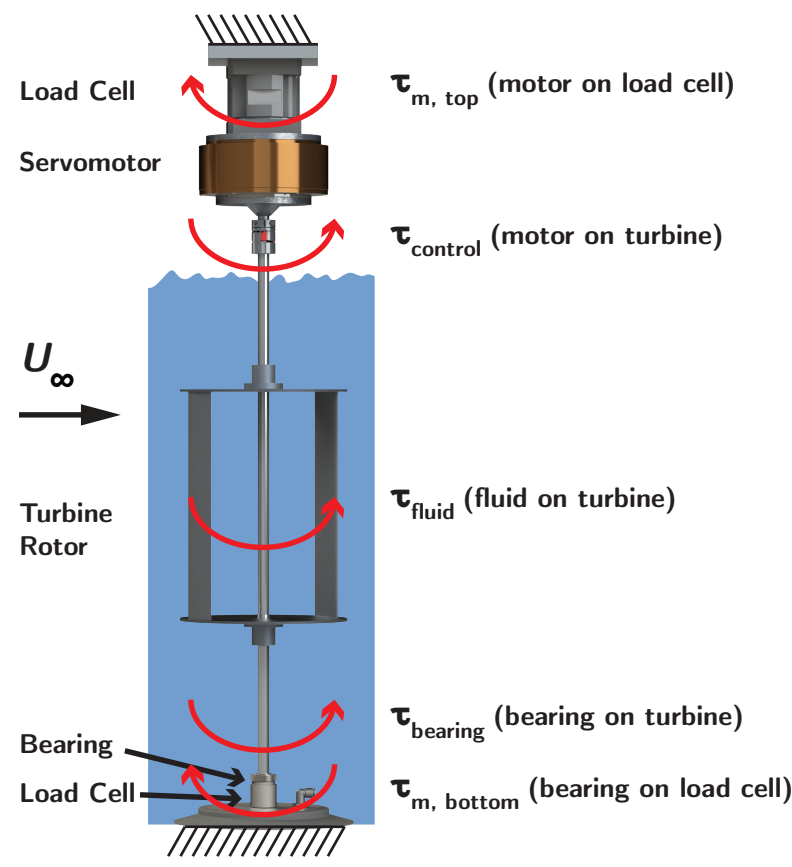

FIG. 1. Free body diagram of the turbine experimental setup showing torques applied to the turbine (shown counterclockwise) and the measured reaction torques (shown clockwise).

we arrive at the net fluid torque on the turbine used to compute $C_{P}$. Positive $\tau$ (torque in the direction of rotation) is produced by lift on the blades while negative $\tau$ is produced by drag on the blades and support structures.

The ten blade connection geometries tested are illustrated in Fig. 2 and listed in Table I. Six types of struts with a chord length equal to the blade chord were tested. These had either a symmetric 4-digit NACA foil, rounded, or rectangular cross-section. For each of these, two thicknesses were tested, $t=0.16 c$ and $0.08 c$, where $c$ is the chord length (equal for the blades and the struts). Additionally, three sets of $0.08 c$ thick disks were tested. The first had a radius equal to that of the turbine. The second and third had radii that were expanded by $a=0.5$ and 1.0 chord lengths. All mounting structures were attached at the ends of the turbine blades, with the exception of a final "mid-span strut" configuration, in which the blades were mounted to the center shaft at the midspan using a $0.16 \mathrm{c}$ thick foil.

By incrementing the tip-speed ratio, a complete performance curve was generated for each mounting structure at four free-stream velocities. These corresponded to blade chord Reynolds numbers,

$$
R e_{c}=\frac{c U_{\infty}}{\nu},
$$

of $18,23,27$ and $32 \times 10^{3}$, where $\nu$ is the kinematic viscosity. Losses due to drag on the mounting structures were estimated by performing the same tip-speed ratio sweeps with the mounting structures alone, after the method of Bachant et al. ${ }^{16}$.

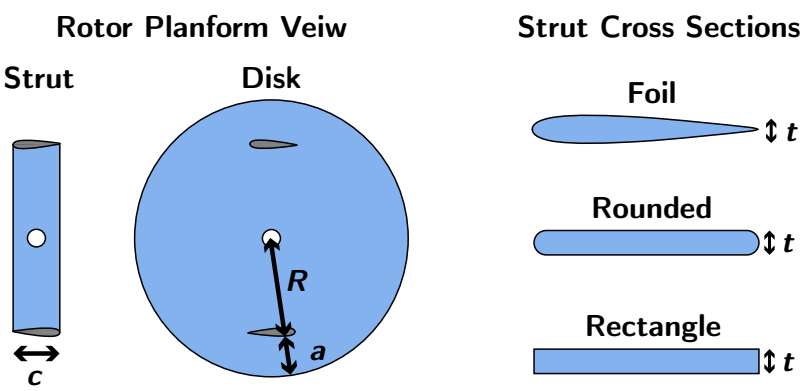

FIG. 2. Blade mounting geometries. Strut versus disk configurations are at the left pane. The three strut cross-sections are shown at the right. Struts and disks are attached to the blade tips, with the exception of the mid-span strut (or "H") configuration, where the blades are mounted via a single foil at the mid-span. Table I lists the mounting geometries tested.

\begin{tabular}{lll}
\hline Planform & Cross Section & Thickness \\
\hline \hline Strut & Foil (NACA0008) & $0.08 c$ \\
\hline Strut & Foil (NACA0016) & $0.16 c$ \\
\hline Strut & Rounded & $0.08 c$ \\
\hline Strut & Rounded & $0.16 c$ \\
\hline Strut & Retangle & $0.08 c$ \\
\hline Strut & Retangle & $0.16 c$ \\
\hline Mid-span strut & Foil (NACA0016) & $0.16 c$ \\
\hline Disk, $R^{*}=R$ & & $0.08 c$ \\
\hline Disk, $R^{*}=R+0.5 c$ & & $0.08 c$ \\
\hline Disk, $R^{*}=R+c$ & & $0.08 c$ \\
\hline
\end{tabular}

TABLE I. List of mounting geometries tested. All geometries except the mid-span strut consist of two mounting structures, one at each end of the rotor. The Mid-span strut is a single mounting structure located at the mid-pane of the rotor. $R^{*}$ indicates disk mounting structure radius.

Uncertainty in the estimation of $C_{P}$ is due primarily to the upper load cell torque and the free stream velocity measurements. The high resolution of the angular encoder and the realatively small values of lower bearing torque mean these measurements do not contribute significant uncertainty. The upper load cell (ATI Mini45, $5 \mathrm{Nm}$ calibration) had an accuracy of $\pm 1 / 1504$ $\mathrm{Nm}$, while the Nortek Vector had an accuracy of $\pm 0.5 \%$ and a resolution of $\pm 0.001 \mathrm{~m} / \mathrm{s}$. The peak $C_{P}$ of the smallest-radius disk mounting structure was 0.198 while the corresponding maximum combined error due to these instruments was $1.3 \times 10^{-4}$.

Over the course of the 30 second measurement period, the number of turbine revolutions ranged from 22 at the lowest tip-speed ratio and Reynolds number to a maximum of 101 revolutions. The cycle-to-cycle variance, as a percent of the mean $C_{P}$ for each data point with an efficiency of $C_{P}>0.05$ (to avoid taking a percentage of near-zero values) was at most $0.38 \%$. The median variance of all performance data points of $C_{P}>0.05$ as a percent of each $C_{P}$ was $0.039 \%$. 


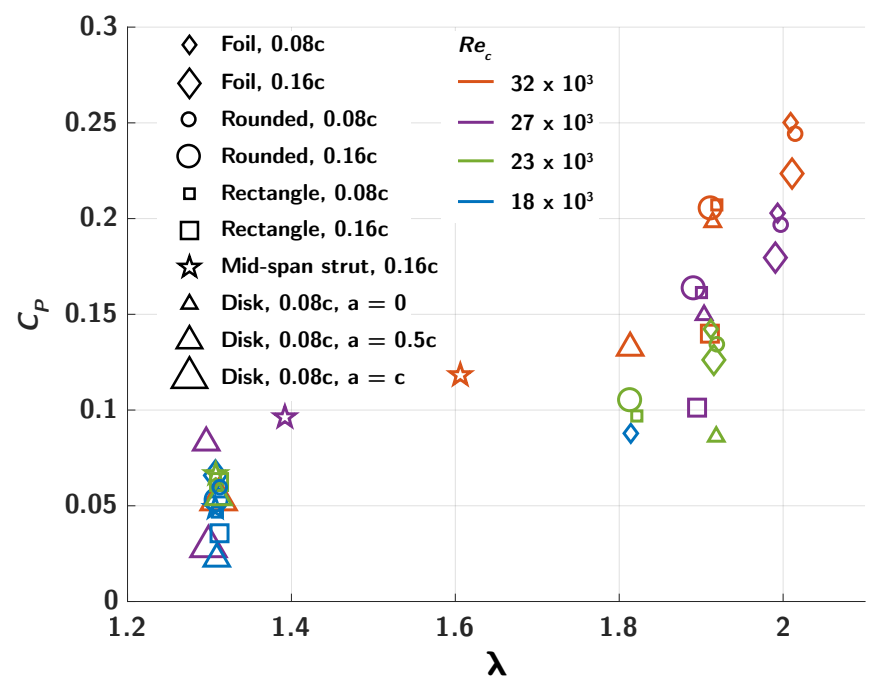

FIG. 3. Peak turbine efficiency as a function of corresponding tip-speed ratio. Color indicates Reynolds number, Eq. (6). Legend indicates strut shape and thickness.

\section{RESULTS}

Figure 3 summarizes the peak performance of each blade mounting geometry as a function of Reynolds number and tip-speed ratio. Without exception, increasing Reynolds number increased turbine performance, as expected for transitional Reynolds numbers ${ }^{16}$. As shown in Fig. 4 (top), for poor-performing turbines, a secondary performance peak at $\lambda=1.3$ dominates over the peak near $\lambda=1.8-2$. Previous work has demonstrated that this low tip-speed ratio peak is due to a strong vortex-foil interaction $^{31}$. Increasing turbine performance generally corresponds to an increase in the tip-speed ratio at which peak efficiency occurs. At the highest Reynolds number, strut geometries with the greatest peak efficiencies were the $0.08 \mathrm{c}$ thick struts with foil and rounded cross sections $\left(C_{P}=0.250\right.$ and 0.244 , respectively) followed by the $0.16 \mathrm{c}$ thick strut with a foil cross section $\left(C_{P}=\right.$ $0.224)$. These geometries performed best at all Reynolds numbers. The smallest disk $(a=0)$, thin rectangular strut, and thick rounded strut had the next best efficiency with similar relative performance at each Reynolds number. The expanded disks $(a=0.5 c, c)$ and the mid-span strut configurations had the poorest performance at each Reynolds number.

\section{DISCUSSION}

Taking a perturbation theory-like approach to the problem similar to $\mathrm{Li}$ and $\mathrm{Calisal}^{30}$, the total efficiency of the turbine may be expressed as follows

$$
C_{P}=C_{P, \mathrm{~b}}+C_{P, \mathrm{~m}}+C_{P, \mathrm{~m} \rightarrow \mathrm{b}}+C_{P, \mathrm{~b} \rightarrow \mathrm{m}}+C_{P, \text { h.o.t. }}
$$

Here $C_{P, \mathrm{~b}}$ represents the ideal power that could be

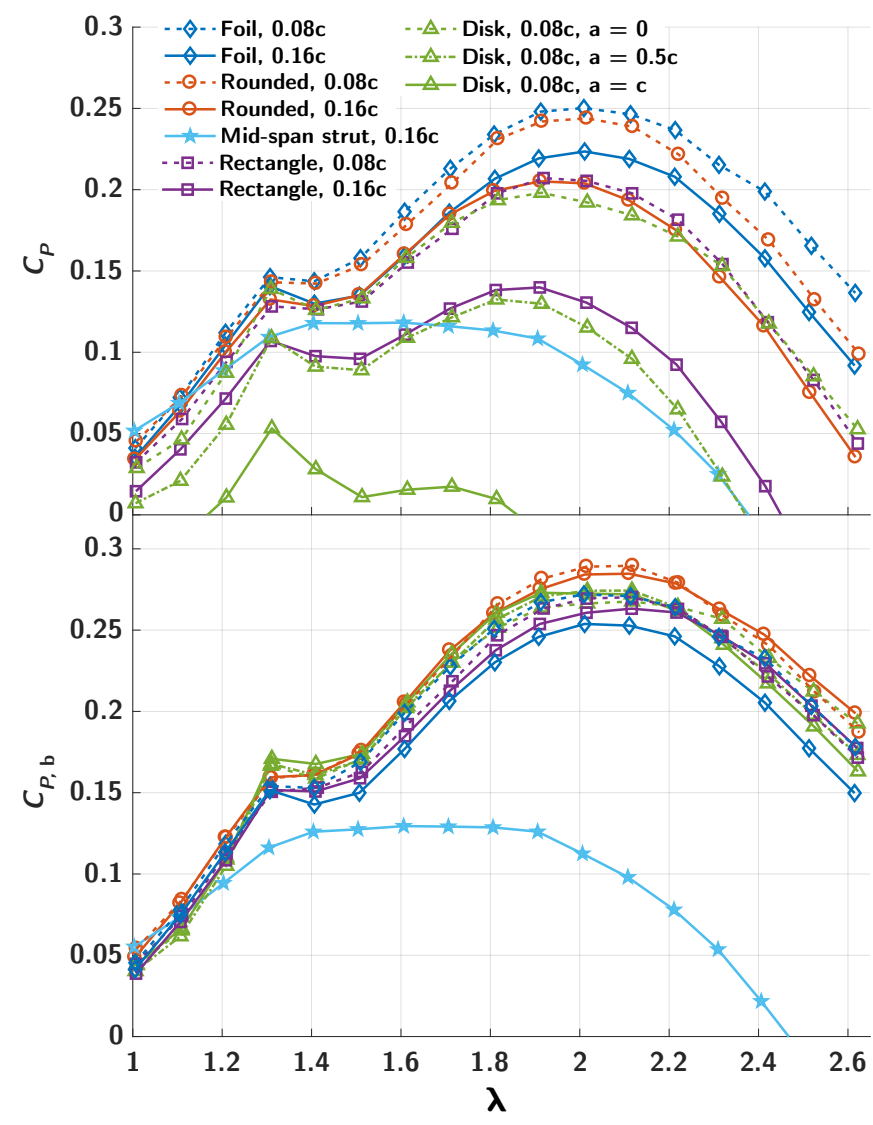

FIG. 4. Top: Full turbine performance curves. Bottom: Performance curves with mounting structure rotational drag losses added back in as an estimate of blade-only performance. Both show performance for $R e_{c}=32 \times 10^{3}$.

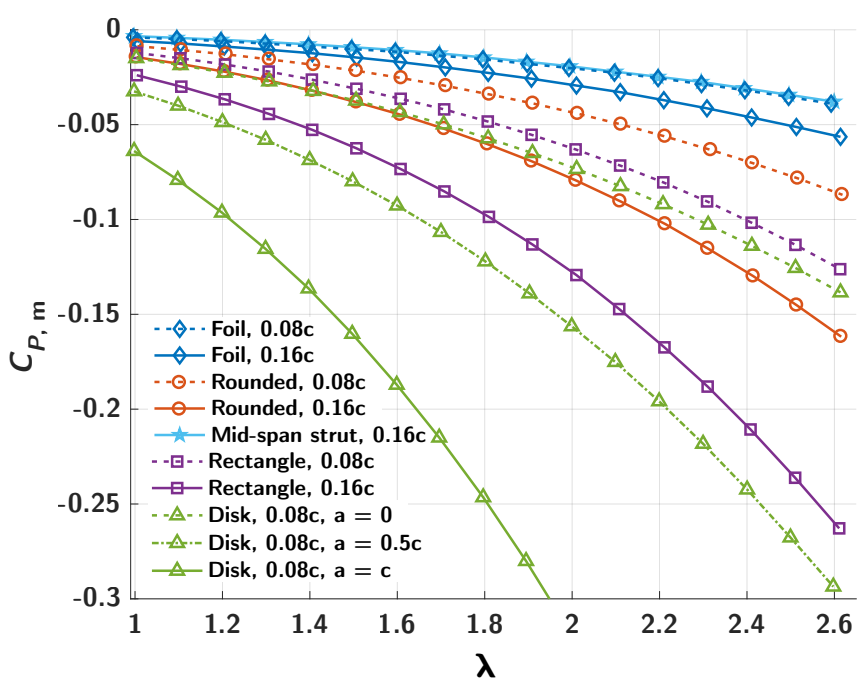

FIG. 5. Efficiency loss due to mounting structure drag versus tip-speed ratio at $R e_{c}=32 \times 10^{3}$. 
produced by the blades, regardless of the mounting structure or blade end condition (e.g., in the absence of tip losses). $C_{P, \mathrm{~m}}$ represents the efficiency losses due to rotational drag on the mounting structures in a flow undisturbed by the turbine blades. The next two terms represent secondary effects. $C_{P, \mathrm{~m} \rightarrow \mathrm{b}}$ represents the influence of the mounting structures on blade performance. This includes a reduction in tip-losses and induced drag and influence on span-wise flow. $C_{P, \mathrm{~b} \rightarrow \mathrm{m}}$ encapsulates the effect of the blades on the drag experienced by the mounting structures, primarily due to changes in the local flow field induced by the blades. Further, higher-order terms $\left(C_{P}\right.$, h.o.t. $)$ likely exist, but are not considered in this analysis.

If secondary and higher-order effects are small enough to be neglected, we can write

$$
C_{P, \mathrm{~b}}=C_{P}-C_{P, \mathrm{~m}}-C_{P, \text { secondary. }} 0
$$

As in Bachant et al. ${ }^{16}$, measurements of $C_{P, \mathrm{~m}}$ have been taken directly by spinning the turbine in the flume without blades. Figure 5 shows this efficiency loss for the highest Reynolds number tested. If the approximation that $C_{P \text {, secondary }} \approx 0$ holds, $C_{P, \mathrm{~b}}$ should be the same for all turbine configurations. Figure 4 (bottom) shows the performance curves resulting from this analysis. With the exception of the mid-span strut turbine, the blade performance curves are generally collapsed, though secondary effects are still responsible for some performance variation. Excluding the mid-span strut turbine, there is a $13 \%$ difference in the lowest and highest performing geometries at the peak of the curves, with discrepancy increasing with tip-speed ratio. The largest variation is between turbines with identical plan-form geometries (Foil, $0.16 c$ versus Rounded 0.08c). These geometries likely have a similar end-plate and winglet effect on the blades, suggesting that $C_{P, \mathrm{~m} \rightarrow \mathrm{b}}$ should be similar. Therefore, it is hypothesized that the most influential secondary effect for all geometries except the mid-span strut geometry is $C_{P, \mathrm{~b} \rightarrow \mathrm{m}}$, that is, the changes in mounting structure drag due to the flow induced by the blades. Extending the disks beyond the radius of the turbine foils did not appear to increase blade performance. This suggests that shielding only the inside edge of the blade (suction side) is adequate to reduce tip losses.

\section{A. Mid-Span Mounting Discussion}

The mid-span strut turbine exhibits a much reduced total performance, remarkable considering mounting structure losses $\left(C_{P, \mathrm{~m}}\right)$ are equal to the best performing turbine (Foil, $0.08 c$, Fig. 5) and that this is a common geometry for small vertical-axis wind turbines (i.e. "H-Darrieus"). When the mounting structure losses are accounted for, the $C_{P, \mathrm{~b}}$ curve has a much broader peak, far below the other turbine geometries (Fig. 4, bottom). Since this turbine is the only geometry with free foil tips,

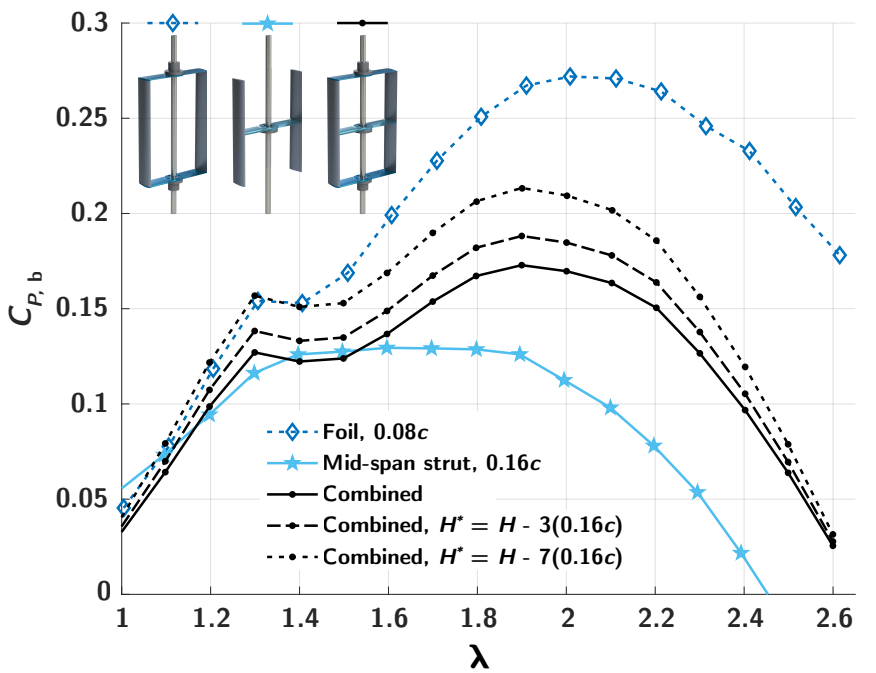

FIG. 6. Blade performance (Eq. (8)) curves for the $0.08 c$ thick foil struts, $0.16 c$ thick mid-span strut, and a turbine with both. Black dashed lines show the performance of the turbine with both mounting structure types using a reduced value of the turbine height in the efficiency equation to account for loss of operable blade span due to the presence of the mid-span strut. Failure of these curves to approach the $0.08 c$ foil curve indicates additional sources of power loss due to the presence of the mid-span strut.

it is likely that $C_{P, \mathrm{~m} \rightarrow \mathrm{b}}$ dominates. Tip-losses are expected to be large as flow is no longer blocked between the suction and pressure sides of the blades. In addition, the free blade tips may introduce additional drag. To examine whether tip-effects are solely responsible for the decrease in performance of the mid-span strut turbine, an additional turbine geometry was tested, where the mounting structure included both the $0.08 \mathrm{c}$ thick foil struts at the ends of the blades and the $0.16 c$ thick foil mid-span strut. A comparison of the foil $0.08 c$, mid-span strut, and the combined turbine are given in Fig. 6. Here, the efficiency loss due to rotational drag, $C_{P} \mathrm{~m}$, has been subtracted, leaving only the blade performance and secondary effects. Shielding the foil tips by using both types of mounting structures increases in blade performance over using the mid-span strut alone. However, the failure of the blade performance to match that achieved using the $0.08 \mathrm{c}$ foil struts alone suggests tip losses are only partially responsible for losses due to the presence of the mid-span mount. Since the mid-span strut attaches to the blades on the suction side of the blades, a central segment of the blades is unavailable for lift production. To explore this possibility, the area used in the denominator of the rotor efficiency equation (2) is reduced from $A=2 R H$ to $A=2 R H^{*}$, where $H^{*}=H-n t$, where $t$ is the mid-span strut thickness, and $n$ is the number of strut thicknesses unavailable for lift production at the mid-span of the blades. As apparent from Fig. 6, rescaling the the blade efficiency in this manner does not account for all of the difference in blade performance be- 
tween the $0.08 c$ foil turbine and the combined turbine, even if three strut widths on either side of the mid-span strut are assumed to be unavailable for lift production (black, fine dashed line).

Given the preceding analysis, it seems that another mechanism, besides losses related to the free blade tips and an interruption of the lifting surface, must be partially responsible for the low performance of the mid-span strut turbine design. One hypothesis stems from the fact that the strut interrupts span-wise flow along the lifting surface. To see how this might be detrimental, first consider that the nominal (local) angle of attack on the foil varies as a function of azimuthal blade position, $\theta$. Neglecting flow induced by the turbine rotor, the nominal angle of attack is

$$
\alpha_{n}(\theta)=-\tan ^{-1}\left(\frac{\sin (\theta)}{\lambda+\cos (\theta)}\right)+\alpha_{p}
$$

where $\alpha_{p}$ is the pitch angle of the blade. For $\lambda=2$, the nominal angle of attack for the upstream portion of the cycle (where the majority of power is generated) varies from less than zero to over 24 degrees. This virtual pitchup maneuver to an angle well above the static stall angle can result in the roll up of a leading edge vortex (LEV), a phenomena known as dynamic stall ${ }^{32}$. The low pressure region in the LEV results in a temporary increase in lift above that possible with a foil at constant angle of attack. As reviewed in $\mathrm{Wu}$, Vakili, and $\mathrm{Wu}^{33}$, span-wise flow in the vortex core helps to stabilize the LEV, resulting in greater lift for longer duration before the vortex is shed. This suggests the introduction of a strut into the midspan of the turbine blades could interrupt span-wise flow, causing premature shedding of the LEV and a reduction in lift.

\section{B. Modeling Mounting Structure Drag}

Analytical models for the drag on various mounting structure geometries may be a useful design tool for providing informed design decisions with limited prototype iteration. Here, models are compared to data collected by rotating the mounting structures without blades. Losses due to rotational drag of the central shaft are not included in the models. Losses due to the central shaft are measured separately by rotating the central shaft in the flow without blades or mounting structures at the appropriate free stream velocity and rotation rate. These small losses are then removed from mounting structure losses.

First the rectangular-planform struts (non-disk mounting structures) are considered. The relative velocity perpendicular to a strut section at a distance $r$ from the rotation axis may be written as

$$
U_{\text {rel }}(r, \theta)=\omega r+U_{\infty} \cos (\theta)+U_{\text {induced }},
$$

where $\theta$ is the blade position and is zero when the blade is traveling directly upstream. $U_{\text {induced }}$ consists of any flow

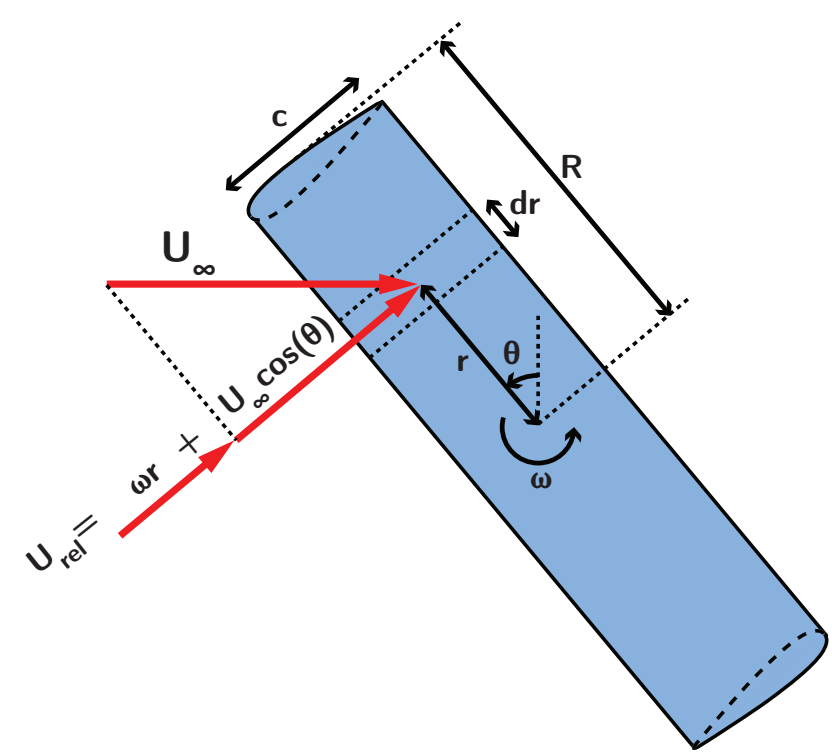

FIG. 7. Strut drag model diagram. The free stream flow is from left to right. Red arrows indicate the components of the local flow velocity due to rotation and the free stream incident on a strut section of width $d r$.

velocities induced by the blades and mounting structures. This term will be neglected for simplicity in subsequent analysis, with the assumption that the flow induced by the mounting structures is small. The resulting velocity vector incident on a strut is shown in Fig. 7. Taking a blade-element approach to calculating strut losses, the torque due to drag on a strut element of radial width $d r$ is

$$
\tau_{d}(r, \theta)=\frac{1}{2} \rho U_{\text {rel }}(r, \theta)^{2} \operatorname{sgn}\left(U_{\text {rel }}\right) C_{D}\left(U_{\text {rel }}\right) L r d r .
$$

Here $L$ is the characteristic length used in the pertinent drag coefficient $\left(C_{D}\right)$ definition (e.g., the chord length for a foil). The $\operatorname{sgn}\left(U_{\text {rel }}\right)$ term ensures the torque is applied in the direction of the relative velocity in case reverse flow is encountered.

At a specific azimuthal angle, $\theta$, the total torque due to drag on one half strut is given by

$$
\tau(\theta)=\frac{1}{2} \rho L \int_{0}^{R} U_{\text {rel }}(r, \theta)^{2} \operatorname{sgn}\left(U_{\text {rel }}\right) C_{D}\left(U_{\text {rel }}\right) r d r
$$

The power loss is the mean of this value over all angles multiplied by the rotation rate, $\omega$, and the number of half struts (twice the number of blades), or

$$
P_{\text {strut }}=\frac{\omega N \rho L}{2 \pi} \int_{0}^{2 \pi} \int_{0}^{R} U_{\text {rel }}(r, \theta)^{2} \operatorname{sgn}\left(U_{\text {rel }}\right) C_{D}\left(U_{\text {rel }}\right) r d r d \theta .
$$




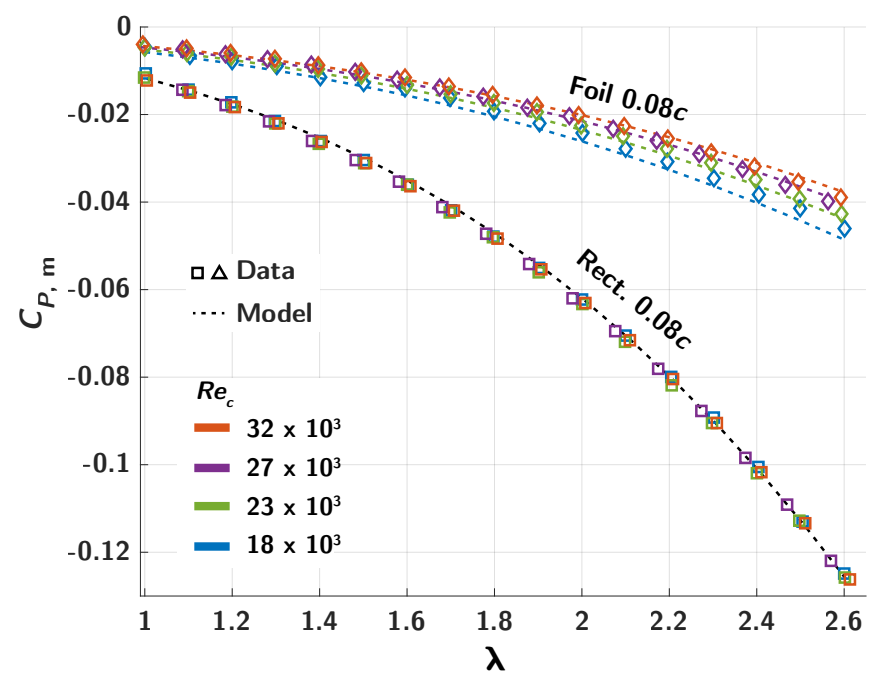

FIG. 8. Efficiency losses due to drag on the $0.08 c$ struts (symbols) for four different Reynolds numbers as measured by rotating the struts without turbine blades. Losses from the center shaft were measured separately and have been accounted for. The $0.08 c$ rectangle line shows predicted losses according to Eq. (14), while the $0.08 c$ foil lines show predicted losses according to Eq. (15), with a modification to account for increased drag of the blade mounting pad.

\section{Rectangular Cross-Section Struts}

Here the length scale used in the drag coefficient is $L=t$ where $t$ is the strut thickness. Because the variation in drag coefficient with Reynolds number has been shown to be small ${ }^{34}$, a constant value is used. A good approximation is to neglect the effect of reverse flow, since it occurs for only a small portion of the rotation, and if the tip-speed ratio is greater than 1 , only near the center of the turbine where $U_{\text {rel }}$ is small. Then the power loss integral can be solved exactly as

$$
P_{\text {rect. }}=\frac{1}{4} \omega N \rho C_{D} t R^{2}\left(\omega^{2} R^{2}+U_{\infty}^{2}\right)
$$

Rectangular sections with a thickness over stream-wise depth ratio of 5 have a drag coefficient of about $C_{D}=$ 1 and drag coefficient trends lower as as this ratio increases $^{35}$. The rectangular struts in this study had width over depth ratios of 6.4 for the $t=0.16 c$ strut and 12.8 for the $t=0.08 c$ strut. Drag coefficients of 0.95 and 0.92 respectively were found to best fit the data, consistent with expectations for an increasing thickness-overdepth ratio. Figure 8 shows the success of this model in predicting the power loss of the $0.08 \mathrm{c}$ rectangular strut, normalized by the fluid power incident on the turbine, as in the standard power coefficient definition. In addition, the assumption of a Reynolds number independent drag coefficient is supported by the independence of power loss from the Reynolds number.

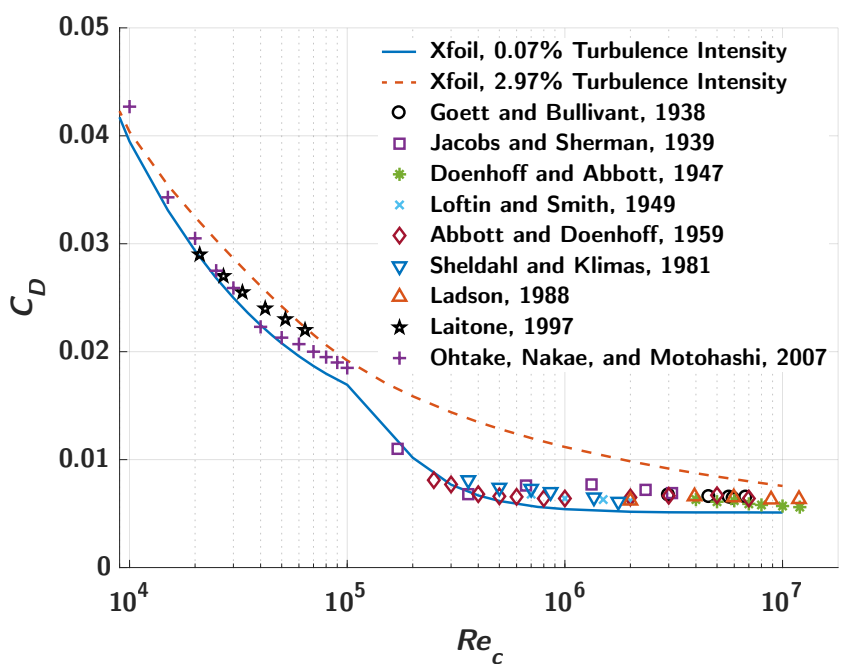

FIG. 9. Drag coefficient versus Reynolds number for a NACA 0012 foil at zero angle of attack. Experimental data, from ${ }^{37-45}$ is compared to the XFOIL code ${ }^{36}$ at two different levels of inflow turbulence intensity. XFOIL is used to generate the drag coefficients in Eq. (15). Low Reynolds number drag is shown to be the worst-case scenario, while high Reynolds number drag coefficients asymptote to a constant value.

\section{Foil Struts}

Equation (13) is applied to model the foil strut power loss. Here the length scale used in the drag coefficient definition is, by convention, the chord length $(L=c)$ and the drag coefficient is allowed to vary based on the instantaneous Reynolds number of each radial element at each angular position, giving

$$
P_{\text {foil }}=\frac{\omega N \rho c}{2 \pi} \int_{0}^{2 \pi} \int_{0}^{R} U_{\text {rel }}(r, \theta)^{2} \operatorname{sgn}\left(U_{\text {rel }}\right) C_{D}(r, \theta) r d r d \theta .
$$

Tabulated values of the drag coefficient variation with Reynolds number were generated using the panel-method code XFOIL $^{36}$ with the turbulence intensity set to $1.5 \%$, the same value as the experiments. These values were interpolated based on the local Reynolds number

$$
R e_{\text {local }}(r, \theta)=\frac{c U_{\text {rel }}(r, \theta)}{\nu}
$$

for each blade element and angular position. XFOIL is able to predict drag coefficients for a NACA0012 foil at zero angle of attack within reasonable accuracy, as shown in Fig. 9.

Because the flat blade mounting area (see Fig. 10) is not a foil in cross section and is exposed while measuring $C_{P, \mathrm{~m}}$, the drag coefficient for this section of the strut $(r / R>0.91)$ was increased by a constant multiple over the foil drag coefficient such that $C_{D}(r, \theta)=$ $c_{3} C_{D \text {, foil }}(r, \theta)$. A constant of $c_{3}=1.68$ was found to best fit the $0.08 c$ foil data. Equation (15) was numerically integrated to arrive at the total power loss. Figure 8 


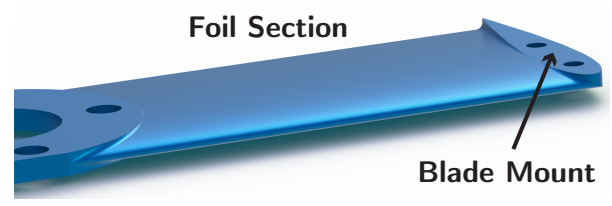

FIG. 10. A close-up of the $0.08 c$ foil strut. The flat blade mount pad adds additional drag when the strut is rotated without blades.

compares this model to data, where losses due to the center shaft have been removed. The Reynolds number dependence of the foil losses are reflected in the analytical model.

\section{Disks}

Von Kármán solved the the Navier Stokes Equations exactly for the flow over a rotating disk with a laminar boundary layer ${ }^{46}$, with refinements later made by Sparrow and Gregg ${ }^{47}$. A drag torque coefficient for a disk exposed to the fluid on both sides, defined as

$$
C_{\tau}=\frac{2 \tau}{\frac{1}{2} \rho \omega^{2} R^{* 5}}
$$

was found to be

$$
C_{\tau}=3.87 R e_{\omega}^{-\frac{1}{2}} .
$$

Here, $R e_{\omega}$ is the disk rotation Reynolds number, given by

$$
R e_{\omega}=\frac{R^{* 2} \omega}{\nu}
$$

where $R^{*}$ is the disk radius. For the extended disk mounting structures $(a>0), R^{*}>R$. Rott and Lewellen ${ }^{48}$ extended the approach to include the translation of the disk, equivalent to introducing a parallel free stream velocity, as in the case considered in these experiments. In the limit of a small advance ratio, defined as

$$
J=\frac{U_{\infty}}{\omega R^{*}},
$$

the solution is identical to Von Kàrmàn's, since the effects on the boundary layer of the free-stream flow cancel on advancing and retreating sides of the disk. Von Kàrmàn also considered the case of a turbulent boundary layer on a rotating disk in still fluid, finding

$$
C_{\tau}=0.146 R e_{\omega}^{-\frac{1}{5}} .
$$

Equations (18) and (21) have shown good agreement with data for disks rotating in a fluid at rest ${ }^{49}$. To determine if the data collected in these experiments lies within the low advance ratio limit, such that these solutions may

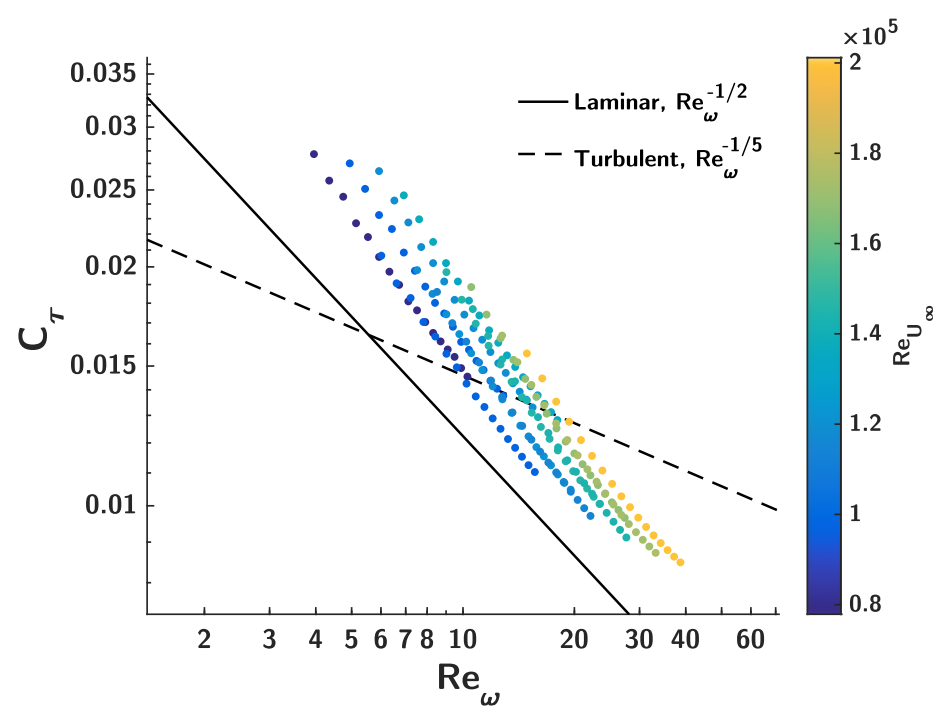

FIG. 11. Disk rotational drag torque coefficient, Eq. (17), data compared to exact solutions for a disk in still fluid with laminar and turbulent boundary layers, Eq. (18) and Eq. $(21)^{46}$.

be used, disk drag torque coefficients measured in these experiments are compared to the exact laminar and turbulent solutions in Fig. 11.

The comparison demonstrates a clear dependence on the free stream velocity, here indicated by the free stream-based Reynolds number,

$$
R e_{U_{\infty}}=\frac{R^{*} U_{\infty}}{\nu}
$$

suggesting that these measurements are above the low advance ratio limit, and cannot rely on the exact solutions alone. Besides that of Rott and Lewellin, the authors are not aware of any research or analysis on the rotational drag on a disk in parallel flow. Figure 11 shows that the drag torque coefficient still approximately follows the $R e_{\omega}^{-\frac{1}{2}}$ law, suggesting the boundary layer is likely in the laminar regime. The slight upward bend in the data with the highest $R e_{\omega}$ may indicate the onset of transition.

To account for the influence of the free stream velocity on the torque coefficient, a linear advance ratio term is added to the laminar solution, such that

$$
C_{\tau}=3.87 R e_{\omega}^{-\frac{1}{2}}+p J
$$

where the constant $p$ was found to be 0.0075 through best fit to the data. This empirical correction term was chosen as it results in the analytical solution for the case that the free stream velocity is zero (or as the advance ratio tends towards infinity). The model is compared to data in Fig. 12, where the predicted power loss has been normalized by the fluid power incident on the turbine rotor. Further measurements would be required to understand if this model is effective across a broader range of Reynolds numbers. 


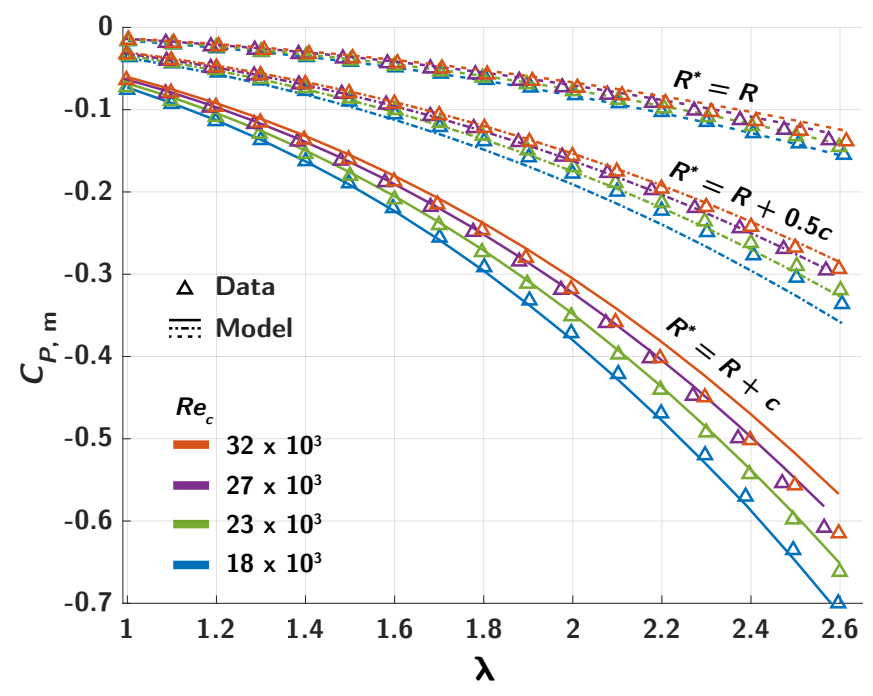

FIG. 12. Efficiency losses due to drag on rotating disks (symbols) for four different Reynolds numbers as measured by rotating the disk without turbine blades. Losses due to the rotation of the center shaft have been removed. The lines show predicted losses according to the drag torque predicted by Eq. (23).

\section{Dependence on Turbine Parameters}

To explore dependence of mounting structure losses on turbine parameters (e.g., number of blades), the models for strut losses are reformulated in terms of nondimensional turbine operational parameters. To aid comparison, some additional assumptions are made. The first of these is that mounting structures are located only at the ends of the blades. Power loss due to the mounting structures is first normalized by the power in the free stream flow incident on the turbine rotor area as

$$
C_{P, \mathrm{~m}}=\frac{P_{\mathrm{m}}}{\frac{1}{2} \rho U_{\infty}^{3} 2 R H} .
$$

Turbine non-dimensional parameters are then substituted. For the rectangular strut model, assuming the strut width is equal the turbine blade chord, this gives

$$
C_{P, \text { rect. }}=-\frac{\pi t^{*} C_{D} \sigma}{4 A_{R}} \lambda\left(\lambda^{2}+1\right)
$$

Here $\sigma$ is the turbine solidity (Eq. (3)),

$$
A_{R}=\frac{H}{2 R}
$$

is the turbine aspect ratio, and

$$
t^{*}=\frac{t}{c}
$$

is the thickness ratio of the rectangular strut sections. For foil struts, if it is assumed that the local Reynolds number is high enough that the drag coefficient is constant, the result is the same as the rectangular strut efficiency loss equation without the thickness ratio due to the difference in drag coefficient definitions:

$$
C_{P, \text { foil }}=-\frac{\pi C_{D} \sigma}{4 A_{R}} \lambda\left(\lambda^{2}+1\right) .
$$

Reynolds number independent operation is likely for commercial-scale turbines, so this simplification is appropriate. For the disk efficiency loss, it is assumed that the disk has the same radius as the turbine blades, $R^{*}=R$, thus the disk advance ratio is equal to inverse of the turbine tip-speed ratio, $J=\frac{1}{\lambda}$. The free stream velocity Reynolds number is a more natural expression for the scale of a turbine, thus the identity

$$
R e_{\omega}=\lambda R e_{U_{\infty}}
$$

is applied to Eq. (23). The turbine efficiency lost to disk drag torque then becomes

$$
C_{P, \text { disk }}=-\frac{\pi}{4 A_{R}} \lambda^{2}\left(3.87 \sqrt{\frac{\lambda}{R e_{U_{\infty}}}}+0.0075\right)
$$

A comparison of Eq.s (25), (28), and (30) shows that for all geometries, increasing the aspect ratio will decrease relative losses, since there is more rotor area per blade mounting structure. A practical upper limit may exist if the blade requires intermediate support for structural stability. For the rectangular and foil struts, losses increase with turbine solidity, while for the disks they do not. However, there is likely a limit to the validity of this dependence on solidity, when the flow induced by a large number of struts has a significant impact on the individual strut drag. The effect of tip-speed ratio is similar across geometries, with the leading term of $\lambda^{3}$ for the foil and rectangular struts and $\lambda^{\frac{5}{2}}$ for the disks. Therefore the choice of mounting geometry (and associated losses) becomes increasingly important at higher tip-speed ratios.

\section{Scaling and Reynolds Number Effects}

A natural question whether the results of this study can generalize to larger geometries and, thus, higher Reynolds numbers, such as those of commercial-scale turbines. Though this topic warrants further exploration, some inferences may be made. First, for the foil and disk mounting geometries, the experimental data here represents a worst-case-scenario for normalized losses. As shown by Fig. 9, foil drag coefficients are expected to drop further as Reynolds number increases. Similarly, the disk losses show an inverse power law dependence on Reynolds number. In contrast, the drag coefficients of rectangular cross section struts show no change with Reynolds number, so those results would likely translate to larger turbines directly. This is because profile drag, 


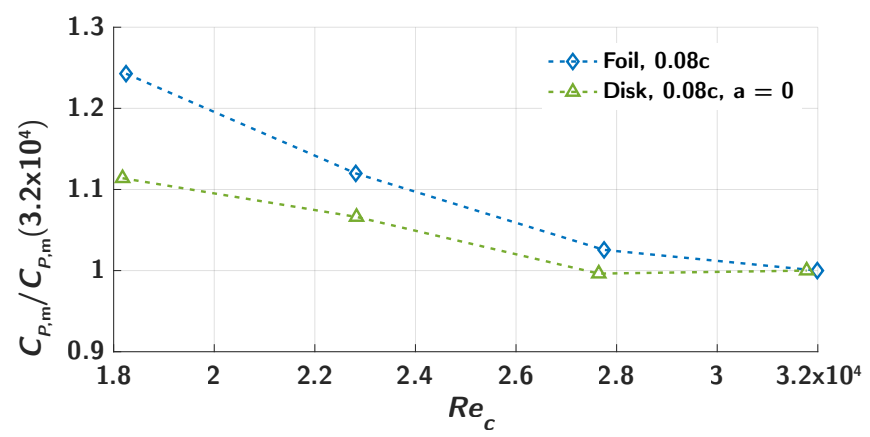

FIG. 13. Efficiency loss as a function of Reynolds number, normalized by the efficiency loss at $R e_{c}=3.2 \times 10^{4}$, is shown for the thin foil and smallest disk mounting geometries. Since the efficiency loss slope is less negative with increase in Reynolds number, these geometries may be approaching Reynolds number independence.

relatively insensitive to Reynolds number for blunt objects, is responsible for the majority of the drag for this mounting geometry.

There is some evidence that these experiments are beginning to approach a regime of Reynolds number insensitivity. Figure 13 shows normalized losses, as compared to the highest Reynolds number measured, appear to be asymptotic to a constant value for the thin foil and smallest disk geometries.

\section{E. Extraploation of Models}

The presented models include terms fit to our specific experimental data. This raises potential concerns over extensions to other turbines and flow conditions. Here, we discuss considerations for extrapolating each model for mounting structure power loss. The model for the rectangular-cross section struts is most likely to remain predictive when applied to different scenarios since the fit value of the drag coefficient is close to that reported in previous work and is Reynolds number independent. The fit value in the foil strut model concerns the drag produced by the sharp geometry of the blade mounting pads. In a fully-assembled turbine, this geometry is merged with the blade, likely reducing the drag produced by the mounting pad. Good estimates of foil strut losses in a full turbine are likely possible using only the XFOIL estimated drag coefficients. Larger uncertainty remains for the accuracy of the disk model for higher free stream velocity Reynolds numbers and future work should include verification or modification of this model to ensure applicability across a wide range of turbine sizes and flow conditions.

\section{v. CONCLUSIONS}

Ten two-bladed cross-flow turbine blade mounting geometries have been tested in a recirculating water flume at four Reynolds numbers. The best-performing mounting system consists of thin, foil-shaped struts attached at the ends of the turbine blades. With the exception of the mid-span strut geometry, blade performance is shown to collapse at peak performance when losses due to strut drag are accounted for. Tip-losses are shown to be only partially responsible for the poor performance of the midspan strut turbine. It is hypothesized that interruption of span-wise flow may cause premature shedding of a beneficial leading edge vortex. Simple models for the drag on rectangular plan-form and disk mounting geometries are explored, and are shown to agree well with experimental data.

We believe these results will be useful to designers of cross-flow turbines. As such the main conclusions of this work are presented in terms of end-plate geometry design decisions. First, supports situated at the blade ends appear to improve performance relative to a mid-span support. For blades supported at the mid-span, the addition of winglets or end-plates to blade tips may reduce tip losses, but will increase drag without adding structural support. Additionally, it appears that mid-span supports have detrimental effects in addition to blade tip losses and rotational drag. For this reason, intermediate span supports should be minimized on turbines with a large axial dimension, $H$, as structural constraints allow, particularly when dynamic stall is an important contributor to turbine power output. Second, for a small number of blades, it appears that streamlined struts rather than disks are optimal. This seems likely to be true for turbines with at least three blades, as multiplying $t=0.08 c$ foil strut drag losses by 1.5 results in less drag than the smallest disk mounting structures tested. If possible, a foil cross-section is optimal, but using a simple-tomanufacture rounded rectangular strut performs nearly as well, as long as the thickness is minimized. For turbines with greater numbers of blades, it is likely that the disk mounting structures will be the optimal mounting geometry, as drag is independent of the number of blades. Since disk drag increases with $R^{* 4}$ and we observed no blade performance improvement by extending disks beyond the blades, the disks should not extend beyond the blades. Extension of the rectangular plan-form mounting structures (struts) beyond the blade radius is also likely to be detrimental. Since the suction side of the turbine blade is already shielded, the additional drag will overwhelm any small increase in blade performance due to further shielding of the blade tip.

Additional mounting structure geometries such as foil struts which incorporate small end-plates or winglets and a curved foil-strut interface are under consideration. If possible, strut geometries should be compared at larger scales, in the Reynolds number independent regime. Differences in performance when the inflow is not perpen- 
dicular to the axis of rotation should be considered, since the disk and strut geometries will likely respond differently to axial flow. Flow measurements inside the turbine rotor could be used to verify the additional sources of mid-span strut losses identified in this study.

\section{ACKNOWLEDGMENTS}

Funding: This work was supported by the US Department of Defense Naval Facilities Engineering Command. Initial explorations of this topic that motivated the present work were carried out by Adam Niblick while he was a student at the University of Washington.

\section{REFERENCES}

${ }^{1}$ F. Balduzzi, A. Bianchini, E. A. Carnevale, L. Ferrari, and S. Magnani, "Feasibility analysis of a Darrieus vertical-axis wind turbine installation in the rooftop of a building," Applied Energy 97, 921-929 (2012).

${ }^{2}$ H. J. Sutherland, D. E. Berg, and T. D. Ashwill, "A retrospective of VAWT technology," Sandia Report No. SAND2012-0304 (2012).

${ }^{3}$ B. Strom, S. L. Brunton, and B. L. Polagye, "Intracycle angular velocity control of cross-flow turbines," Nature Energy (accepted, forthcoming) (2017).

${ }^{4}$ J. O. Dabiri, "Potential order-of-magnitude enhancement of wind farm power density via counter-rotating vertical-axis wind turbine arrays," Journal of Renewable and Sustainable Energy 3, 043104 (2011).

${ }^{5}$ J. Abraham, B. Plourde, G. Mowry, W. Minkowycz, and E. Sparrow, "Summary of savonius wind turbine development and future applications for small-scale power generation," Journal of Renewable and Sustainable Energy 4, 042703 (2012).

${ }^{6}$ B. Plourde, J. Abraham, G. Mowry, and W. Minkowycz, "An experimental investigation of a large, vertical-axis wind turbine: Effects of venting and capping," Wind Engineering 35, 213-222 (2011).

${ }^{7}$ S. Salter, "Are nearly all tidal stream turbine designs wrong?" in 4th International Conference on Ocean Energy (2012).

${ }^{8}$ A. Copping, N. Sather, L. Hanna, J. Whiting, G. Zydlewski, G. Staines, A. Gill, I. Hutchison, A. OâHagan, T. Simas, et al., "Annex iv 2016 state of the science report: Environmental effects of marine renewable energy development around the world," Ocean Energy Systems (2016).

${ }^{9}$ T. J. Carrigan, B. H. Dennis, Z. X. Han, and B. P. Wang, "Aerodynamic shape optimization of a vertical-axis wind turbine using differential evolution," ISRN Renewable Energy 2012 (2012).

${ }^{10} \mathrm{M}$. R. Castelli and E. Benini, "Effect of blade inclination angle on a darrieus wind turbine," Journal of turbomachinery 134, 031016 (2012).

${ }^{11}$ C.-C. Chen and C.-H. Kuo, "Effects of pitch angle and blade camber on flow characteristics and performance of small-size darrieus vawt," Journal of Visualization 16, 65-74 (2013).

${ }^{12}$ C. Consul, R. Willden, E. Ferrer, and M. McCulloch, "Influence of solidity on the performance of a cross-flow turbine," in 8th European Wave and Tidal Energy Conference. Uppsala, Sweden (2009).

${ }^{13}$ R. Gosselin, G. Dumas, and M. Boudreau, "Parametric study of h-darrieus vertical-axis turbines using urans simulations," 21st Annual Conference of the CFD Society of Canada (2013).

${ }^{14}$ L. A. Danao, J. Edwards, O. Eboibi, and R. Howell, "A numerical investigation into the effects of fluctuating wind on the performance of a small scale vertical axis wind turbine," Engineering Letters 21, 149-157 (2013).

${ }^{15} \mathrm{D}$. Malcolm, "Dynamic response of a darrieus rotor wind turbine subject to turbulent flow," Engineering Structures 10, 125-134 (1988).

${ }^{16}$ P. Bachant, M. Wosnik, B. Gunawan, and V. S. Neary, "Experimental study of a reference model vertical-axis cross-flow turbine," PloS one 11, e0163799 (2016).

${ }^{17}$ L. Battisti, L. Zanne, S. DellâAnna, V. Dossena, G. Persico, and B. Paradiso, "Aerodynamic measurements on a vertical axis wind turbine in a large scale wind tunnel," Journal of energy resources technology 133, 031201 (2011).

${ }^{18}$ M. Kinzel, Q. Mulligan, and J. O. Dabiri, "Energy exchange in an array of vertical-axis wind turbines," Journal of Turbulence 13, 1-13 (2012).

${ }^{19}$ E. Reid, "The effects of shielding the tips of airfoils", naca rep. 201," Natl. Advis. Comm. Aeronaut., Hampton, VA (1924).

${ }^{20}$ I. Kroo, "Drag due to lift: concepts for prediction and reduction," Annual Review of Fluid Mechanics 33, 587-617 (2001).

${ }^{21} \mathrm{~A}$. Gorlov, "Development of the helical reaction hydraulic turbine. final technical report, july 1, 1996-june 30, 1998," Tech. Rep. (Northeastern Univ., Boston, MA (United States), 1998).

${ }^{22}$ M. Khan, G. Bhuyan, M. Iqbal, and J. Quaicoe, "Hydrokinetic energy conversion systems and assessment of horizontal and vertical axis turbines for river and tidal applications: A technology status review," Applied Energy 86, 1823-1835 (2009).

${ }^{23}$ M. Shiono, K. Suzuki, and S. Kiho, "An experimental study of the characteristics of a darrieus turbine for tidal power generation," Electrical Engineering in Japan 132, 38-47 (2000).

${ }^{24} \mathrm{P}$. Bachant and M. Wosnik, "Performance measurements of cylindrical-and spherical-helical cross-flow marine hydrokinetic turbines, with estimates of exergy efficiency," Renewable Energy 74, 318-325 (2015).

${ }^{25} \mathrm{P}$. Bachant and M. Wosnik, "Characterising the near-wake of a cross-flow turbine," Journal of Turbulence 16, 392-410 (2015).

${ }^{26}$ C. Hill, V. S. Neary, B. Gunawan, M. Guala, and F. Sotiropoulos, "Us department of energy reference model program rm2: Experimental results," Sandia National Laboratories, Albuquerque, NM (2014).

${ }^{27}$ A. Goude, S. Lundin, and M. Leijon, "A parameter study of the influence of struts on the performance of a vertical-axis marine current turbine," in Proceedings of the 8th European wave and tidal energy conference, EWTEC09, Uppsala, Sweden (Citeseer, 2009) pp. 477-483.

${ }^{28}$ R. Gosselin, G. Dumas, and M. Boudreau, "Parametric study of h-darrieus vertical-axis turbines using cfd simulations," Journal of Renewable and Sustainable Energy 8, 053301 (2016).

${ }^{29}$ G. Rawlings, M. Alidadi, V. Klaptocz, Y. Nabavi, Y. Li, J. Mikkelsen, S. Calisal, et al., "Application of end plates for vertical axis hydro turbine performance enhancement," in The Eighteenth International Offshore and Polar Engineering Conference (International Society of Offshore and Polar Engineers, 2008).

${ }^{30}$ Y. Li and S. M. Calisal, "Three-dimensional effects and arm effects on modeling a vertical axis tidal current turbine," Renewable energy 35, 2325-2334 (2010).

${ }^{31}$ B. Strom, S. L. Brunton, and B. Polagye, "Consequences of preset pitch angle on cross-flow turbine hydrodynamics," Proceeding of the European Wave and Tidal Energy Conference (2015).

${ }^{32}$ W. McCroskey, "The phenomenon of dynamic stall." Tech. Rep. (National Aeronautics and Space Administration Moffett Field CA, AMES Research Center, 1981).

${ }^{33} \mathrm{~J}$. Wu, A. Vakili, and J. Wu, "Review of the physics of enhancing vortex lift by unsteady excitation," Progress in Aerospace Sciences 28, 73-131 (1991).

${ }^{34} \mathrm{G}$. Schewe, "Reynolds-number-effects in flow around a rectangular cylinder with aspect ratio 1: 5," Journal of Fluids and Structures 39, 15-26 (2013).

${ }^{35}$ A. Šoda, C. Mannini, and M. Sjerić, "Investigation of unsteady air flow around two-dimensional rectangular cylinders." Transac- 
tions of FAMENA 35 (2011)

${ }^{36}$ M. Drela, "Xfoil: An analysis and design system for low reynolds number airfoils," in Low Reynolds number aerodynamics (Springer, 1989) pp. 1-12.

${ }^{37}$ H. J. Goett and W. K. Bullivant, "Tests of naca 0009, 0012, and 0018 airfoils in the full-scale tunnel, report no.647," Tech. Rep. (National Advisory Commitee for Aeronautics, 1939).

${ }^{38}$ E. N. Jacobs and A. Sherman, "Airfoil section characteristics as affected by variations of the reynolds number, report no. 586," Tech. Rep. (National Advisory Commitee for Aeronautics, 1937).

${ }^{39}$ A. E. Von Doenhoff and F. T. Abbott, "The langley twodimensional low-turbulence pressure tunnel, technical note no. 1283," Tech. Rep. (National Advisory Commitee for Aeronautics, 1947).

${ }^{40}$ L. K. Loftin Jr and H. A. Smith, "Aerodynamic characteristics of 15 naca airfoil sections at seven reynolds numbers from $0.7 \times$ $10^{6}$ to $9.0 \times 10^{6}$, technidal note no. 1945 ," Tech. Rep. (National Advisory Commitee for Aeronautic, 1949).

${ }^{41}$ I. H. Abbott and A. E. Von Doenhoff, Theory of wing sections, including a summary of airfoil data (Courier Corporation, 1959).

${ }^{42}$ R. E. Sheldahl and P. C. Klimas, "Aerodynamic characteristics of seven symmetrical airfoil sections through 180-degree angle of attack for use in aerodynamic analysis of vertical axis wind turbines," Tech. Rep. (Sandia National Labs., Albuquerque, NM
(USA), 1981)

${ }^{43}$ C. L. Ladson, "Effects of independent variation of mach and reynolds numbers on the low-speed aerodynamic characteristics of the naca 0012 airfoil section, nasa technical memorandum 4074," Tech. Rep. (NAtional Aeronautics and Space Administration, 1988).

${ }^{44} \mathrm{E}$. Laitone, "Wind tunnel tests of wings at reynolds numbers below 70 000," Experiments in Fluids 23, 405-409 (1997).

${ }^{45}$ T. Ohtake, Y. Nakae, and T. Motohashi, "Nonlinearity of the aerodynamic characteristics of naca0012 aerofoil at low reynolds numbers," Japan Society of Aeronautical Space Sciences 55, 439445 (2007).

${ }^{46}$ T. Von Kármán, "Über laminare und turbulente reibung," ZAMM - Journal of Applied Mathematics and Mechanics / Zeitschrift für Angewandte Mathematik und Mechanik 1, 233252 (1921).

${ }^{47}$ E. Sparrow and J. Gregg, "Mass transfer, flow, and heat transfer about a rotating disk," Journal of Heat Transfer 82, 294-302 (1960).

${ }^{48}$ N. Rott and W. S. Lewellen, "Boundary layers due to the combined effects of rotation and translation," The Physics of Fluids 10, 1867-1873 (1967).

${ }^{49}$ H. Schlichting, Boundary-layer theory, 7th ed. (McGraw-Hill, 1979). 